\title{
Atomes froids : réseaux optiques et horloges
}

Michèle Leduc ${ }^{(1)}$ (michèle.leduc@lkb.ens.fr) et Pierre Lemonde(2) (pierre.lemonde@obspm.fr)

(1) Laboratoire Kastler Brossel, École Normale Supérieure, 24 rue Lhomond, 75231 Paris Cedex 05

(2) SYRTE, Observatoire de Paris, 61 avenue de l'Observatoire, 75014 Paris

Le domaine des « atomes

froids » est en expansion.

L'interaction lumière-matière

permet de refroidir un gaz

en dessous du microkelvin

et de le piéger dans le vide.

À partir des condensats

de Bose-Einstein, on extrait

des « lasers à atomes ».

On sait aussi faire varier

l'interaction entre les particu-

les. Piégés dans des réseaux

optiques avec des ondes

stationnaires, les atomes

ultra-froids, en particulier

fermioniques, fournissent

des systèmes modèles pour

la physique de la matière

condensée.

L'utilisation d'atomes froids

pour les horloges a permis

d'obtenir un gain considérable

sur l'exactitude, offrant des

possibilités fascinantes de tests

de physique fondamentale.

Une nouvelle révolution

s'annonce avec les horloges

optiques.
Le domaine de recherche autour des atomes refroidis par laser a bientôt trente ans ; aujourd'hui il est en pleine expansion, abordant des territoires toujours nouveaux de la physique fondamentale comme des applications. Le principe du refroidissement et du piégeage des atomes par laser a fait l'objet du $\mathrm{n}^{\circ} 112$ du Bulletin de la SFP (décembre 1997-janvier 1998), après l'attribution du prix Nobel en 1997 à Claude Cohen-Tannoudji, ainsi qu'à ses deux collègues américains Steven Chu et William Phillips. Après un bref rappel des méthodes à la base de ce domaine, le présent article se propose de donner quelques exemples de nouvelles orientations prises parla recherche utilisant des " atomes froids ", sans vouloir couvrir l'ensemble de ce sujet devenu très vaste.

\section{Condensation de Bose-Einstein et lasers à atomes}

Le ralentissement d'un atome par un faisceau laser est fondé sur des cycles d'absorption/émission de photons par l'atome et sur les transferts d'impulsion associés (voir encadré 1, haut) : après un grand nombre de tels cycles, l'atome est fortement freiné dans la direction opposée au faisceau laser, sa vitesse passant de quelques centaines de $\mathrm{m} / \mathrm{s}$ à quelques $\mathrm{cm} / \mathrm{s}$. On peut en outre, avec une méthode semblable, diminuer la température du gaz en réduisant la dispersion de la vitesse des atomes (voir encadré 1, bas). Les températures atteintes se situent dans la zone des microkelvins $(\mu \mathrm{K})$.

Les gaz ultra-froids sont produits dans des pièges qui maintiennent quelques milliards d'atomes dans le vide, loin des parois des enceintes. Le dispositif devenu standard est le piège magnéto-optique ${ }^{(1)}$, utilisé pour toutes sortes d'applications, allant de la métrologie à la chimie froide.
Pourtant, il a fallu développer d'autres types de pièges, purement magnétiques ou purement optiques, pour amener, par une technique dite d' "évaporation" des gaz, jusqu'à des températures proches du zéro absolu (fig. 1). On est ainsi parvenu à mettre en évidence la condensation de BoseEinstein, ce phénomène très étonnant qui consiste à accumuler des bosons dans l'état fondamental d'un piège. La transition de phase de cette condensation se produit lorsque la longueur d'onde de de Broglie associée à chaque particule (qui est inversement proportionnelle à la racine carrée de la température) devient de l'ordre de la distance entre les particules; les paquets d'ondes se recouvrent et une fonction d'onde macroscopique se construit. Le gaz devient ainsi superfluide, comme l'atteste la possibilité d'y engendrer des tourbillons quantiques. La figure 2 montre un des premiers condensats, obtenu avec du sodium par W. Ketterle en 1999 au MIT. Depuis, ce phénomène donne lieu à des développements extraordinaires et la galerie des atomes condensés s'accroît chaque année.

Un exemple d'utilisation des condensats de Bose-Einstein est la création des "lasers à atomes ": on crée une "fuite " dans le condensat, des atomes quittent physiquement le piège et tombent alors sous l'effet de la pesanteur, tout en gardant les propriétés qu'ils avaient dans le condensat. Un paquet d'ondes cohérent de matière se propage ainsi dans l'espace avec des atomes ayant tous la même direction et la même vitesse, présentant beaucoup d'analogies avec un laser optique (voir l'image d'ouverture de la troisième partie, p. 41). Les lasers à atomes peuvent être manipulés, c'est-à-dire réfléchis, focalisés ou séparés en plusieurs bras comme des faisceaux optiques. Leurs applications sont encore à imaginer, même si leur utilisation est déjà envisagée dans de futures générations de gyroscopes atomiques [1]. 

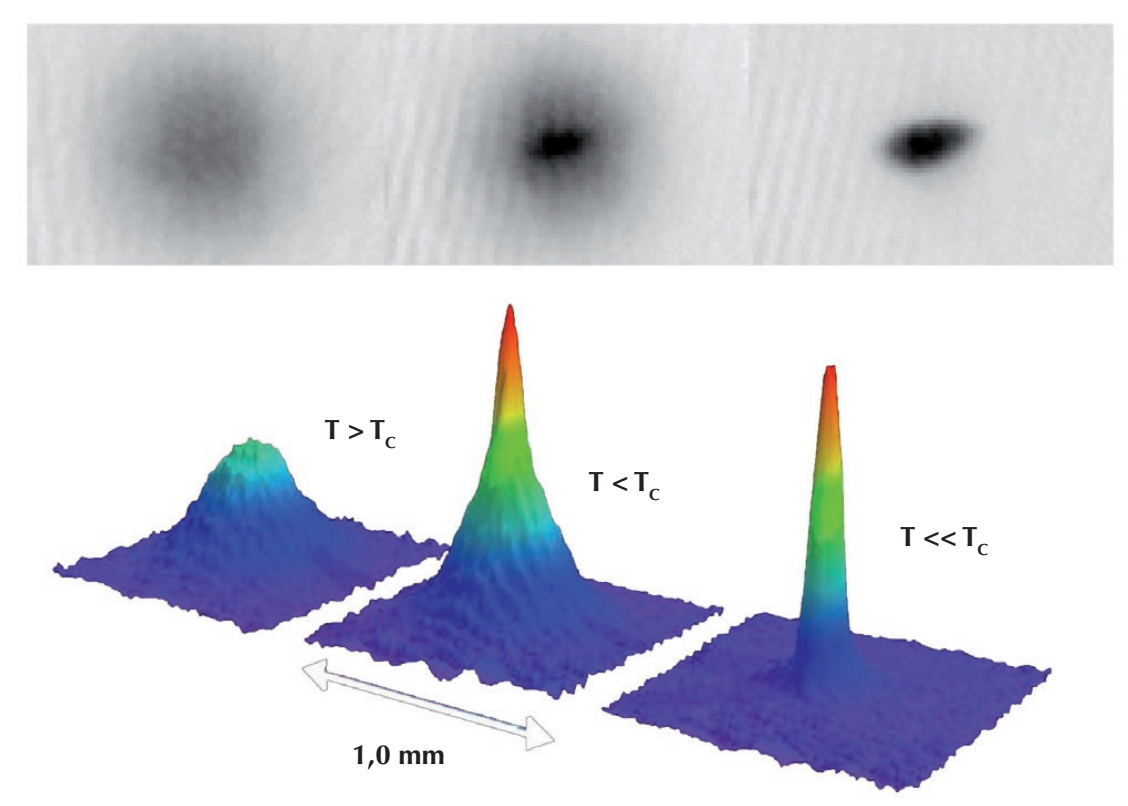

2. Condensation de Bose-Einstein du sodium.

En haut : image d'absorption d'un faisceau sonde par les atomes après expansion du nuage pendant un certain temps de chute libre (mesure dite par temps de vol). On reconstitue ainsi la répartition spatiale des vitesses des atomes dans le condensat initial, trop petit pour être visualisé directement.

En bas : à gauche, au-dessus de la température critique $\mathrm{T}_{\mathrm{C}}$, la distribution des vitesses est celle d'un gaz normal en équilibre thermique; au centre, juste en dessous de $\mathrm{T}_{\mathrm{c}}$, des atomes condensés apparaissent, caractérisés par une seule vitesse (pic étroit au-dessus du fond thermique), mais du gaz non condensé subsiste ; à droite, pratiquement tout le gaz est condensé. (Courtoisie W. Ketterle, MIT-Harvard.)

\section{Résonance de Feshbach}

Encadré 2

La longueur de diffusion a est le paramètre qui, en première approximation, caractérise les collisions entre deux particules à très basse énergie ; par convention, elle est comptée positivement pour une interaction répulsive, et négativement dans le cas opposé. La section efficace $\sigma$ s'écrit $\sigma=4 \pi a^{2}$ pour des particules discernables, $\sigma=8 \pi a^{2}$ pour des bosons identiques et $\sigma=0$ pour des fermions identiques.

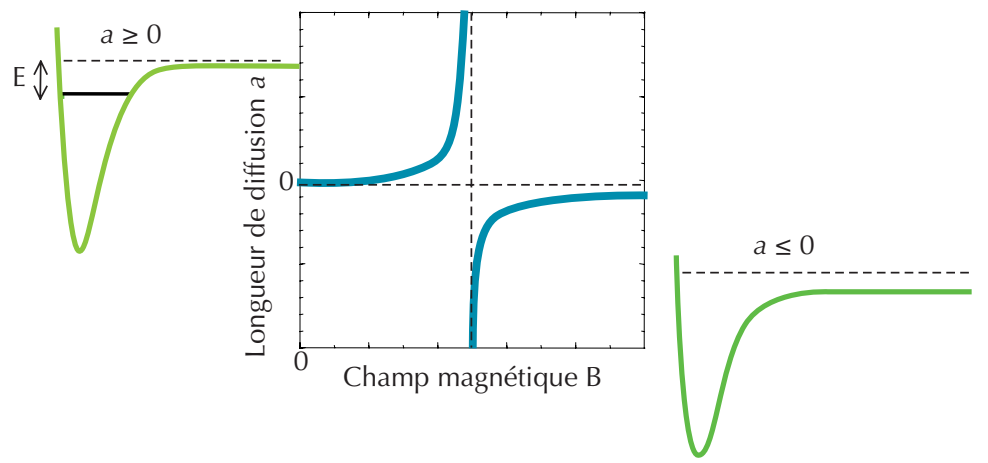

La figure montre au centre comment varie la longueur de diffusion a en fonction du champ magnétique B lors d'une résonance de Feshbach. En vert, figure le potentiel $d^{\prime}$ interaction entre deux atomes froids en fonction de leur distance. À courte distance, les deux atomes peuvent former une molécule dimère aux états d'énergie quantifiés. On voit (potentiel à gauche) l'état moléculaire le moins lié dans le potentiel d'interaction dont l'énergie de liaison, en première approximation, est $\mathrm{E}=\hbar^{2} / \mathrm{ma}^{2}$. Si I'on applique un champ magnétique, on déplace la position de ce dernier niveau moléculaire dans le potentiel ; lorsqu'il s'approche du seuil de dissociation, c'est-à-dire que E devient très petit, la longueur de diffusion a augmente considérablement et tend vers l'infini.

\section{\〉}

(voir encadré 2). Appliquée à un condensat de Bose-Einstein, où $a$ est positif, la traversée d'une résonance de Feshbach est susceptible de faire exploser le condensat. Mais c'est pour les gaz fermioniques que ces résonances présentent le plus grand intérêt : en effet, en s'approchant très près de la résonance du côté où $a$ est négatif, on produit une attraction si forte entre deux fermions, qu'on réussit à les lier dans une molécule dont la durée de vie peut atteindre plusieurs secondes. De tels dimères de fermions constituent des bosons composites, qui sont alors susceptibles de donner lieu à un condensat de Bose-Einstein, pourvu que leur densité soit suffisante. Ces condensats moléculaires sont maintenant couramment étudiés.

Si l'on traverse la résonance de Feshbach pour se retrouver du côté où $a$ est positif, d'autres phénomènes quantiques très intéressants se produisent : les fermions sont susceptibles de s'apparier en formant des paires très lâches d'atomes de moments angulaires opposés, qui présentent beaucoup d'analogies avec les paires de Cooper d'électrons dans les matériaux supraconducteurs. La transition entre la condensation de molécules dimères et la formation de paires du type " paires de Cooper " fait l'objet de recherches très actives, tant sur le plan expérimental que théorique.

\section{Des atomes froids dans des réseaux optiques}

Les gaz d'atomes ultra-froids, bosoniques ou fermioniques, sont des systèmes robustes et versatiles qui servent aujourd'hui à étudier de nombreux problèmes fondamentaux. Nous venons de voir avec les "paires de Cooper " un exemple où les atomes froids fournissent un système modèle. D'autres exemples sont maintenant fournis par des atomes froids distribués dans des structures régulières dans l'espace. Pour cela, on piège les atomes dans des potentiels périodiques formés de réseaux optiques. Les atomes sont alors confinés, non plus dans des pièges magnétiques, mais dans des pièges optiques dipolaires fondés sur l'interaction entre le moment dipolaire induit d'un atome et un champ électrique extérieur, tel que le champ oscillant d'un laser. La fréquence du laser est en général choisie loin de la résonance atomique, de façon à minimiser l'émission/ absorption du laser par les atomes. En superposant des faisceaux se propageant en sens inverse, on forme une onde stationnaire dont les nœuds ou les ventres 
constituent un réseau périodique de puits de potentiel. Avec plusieurs paires de lasers, on peut obtenir des réseaux périodiques de dimension 1D, 2D ou 3D. Un réseau 1D (une seule onde stationnaire) produit un ensemble de disques d'atomes froids ; un réseau 2D (deux ondes stationnaires) confine les atomes dans des tubes minces comme des aiguilles (fig. 3a) ; en 3D (trois ondes stationnaires), ils sont localisés comme aux sommets des mailles d'un cristal cubique (fig. 3b). De tels agencements artificiels d'atomes peuvent être bien plus variés que ceux des matériaux solides, et surtout on peut à volonté en manipuler les paramètres : forme et dimensionnalité du réseau, taille de la maille, force de l'interaction entre les particules, etc. On dispose ainsi d'un système quantique hautement contrôlable, pour simuler les propriétés dynamiques d'autres systèmes quantiques beaucoup plus complexes, tels que ceux observés en matière condensée.

Un autre avantage de tels réseaux est qu'on peut faire varier la profondeur des puits avec l'intensité du laser et jouer avec les interactions entre les particules, qui se font sentir d'un site à l'autre. Si ces interactions deviennent plus fortes que l'énergie cinétique, les états quantiques deviennent fortement corrélés, une situation extrêmement intéressante pour la théorie de la matière condensée. Un exemple de transition entre un système quantique en interaction faible et un système fortement corrélé à $n$-corps est donné par la transition superfluideisolant de Mott $^{(3)}$, observée avec des bosons condensés lorsqu'on augmente la profondeur des puits du réseau optique. Lorsque les puits sont peu profonds, le gaz demeure superfluide et peut être décrit par une fonction d'onde géante étendue sur tout le réseau ; les atomes ne sont pas localisés dans un site donné et circulent à l'aise d'un site à l'autre par effet tunnel. Au contraire, au-delà d'un certain seuil le système "se prend en masse ", les atomes se localisent à un ou plusieurs par site, la superfluidité ainsi que la cohérence d'ensemble disparaissent. Ceci est illustré sur la figure 4. Comprendre la dynamique de ces transitions pose beaucoup de questions. D'autres ouvertures vers la physique de la matière désordonnée et la localisation d'Anderson sont également offertes par les atomes froids dans les réseaux optiques. Toutes ces recherches en sont encore à leurs débuts.
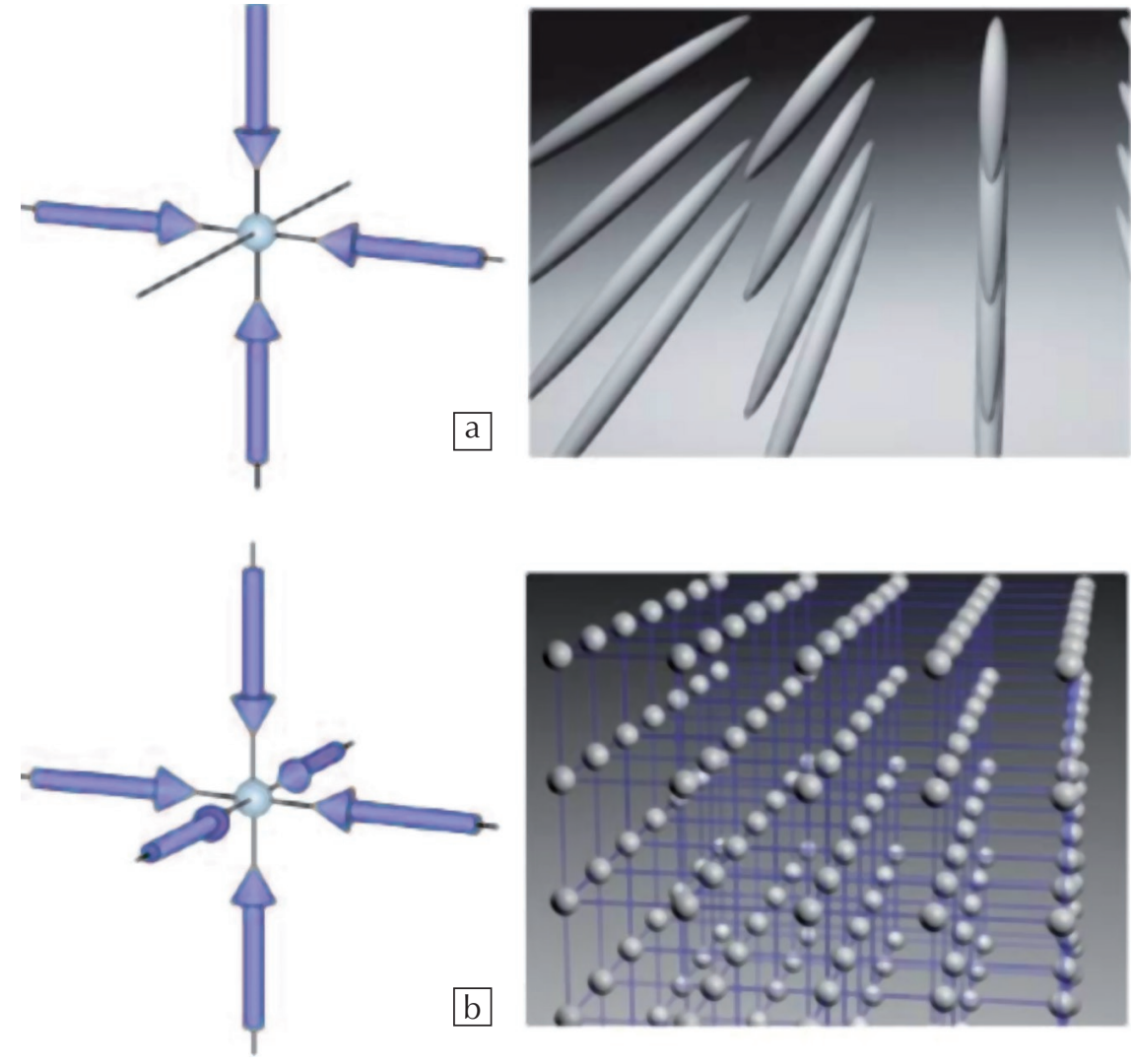

3. Atomes froids piégés dans des réseaux optiques. Des paires de faisceaux laser (flèches bleues) formant des ondes stationnaires créent des potentiels périodiques électromagnétiques dans lesquels les atomes viennent se piéger. En (a) réseau à deux dimensions, en (b) réseau à trois dimensions.

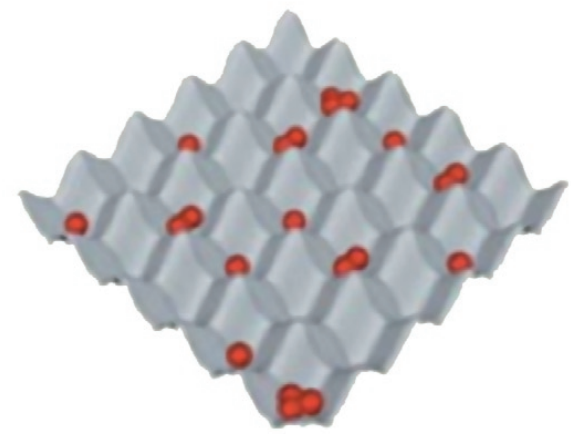

$\mathrm{a}$

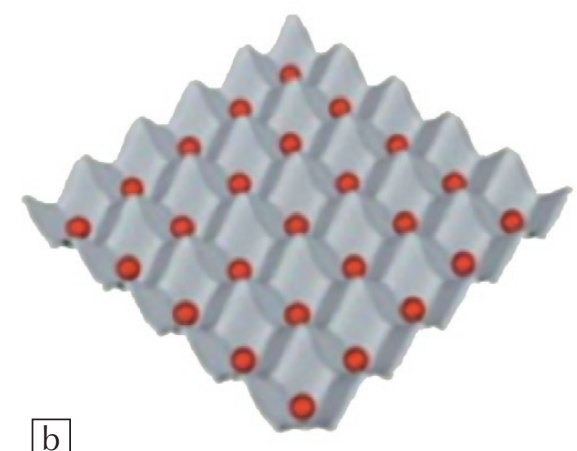

b

4. Transition superfluide/isolant de Mott pour des condensats de Bose-Einstein dans un réseau optique en trois dimensions (vision d'artiste). On passe de (a) à (b) en faisant varier l'intensité du faisceau laser qui piège les atomes dans le réseau. En (a) les puits de potentiel du réseau sont peu profonds, les atomes circulent librement entre les sites par effet tunnel, la fonction d'onde macroscopique s'étend sur tout le réseau, le gaz a des propriétés superfluides. En (b) la profondeur des puits de potentiel est grande, la position des atomes est fixée dans le réseau, la superfluidité a disparu. 


\section{Des horloges toujours plus précises}

Si les atomes froids permettent des progrès spectaculaires dans certains domaines de la physique fondamentale, leur intérêt ne se limite pas à cela et ils trouvent des applications pratiques de plus en plus nombreuses. La plus importante concerne les horloges atomiques. Depuis une cinquantaine d'années, les meilleures horloges sont basées sur la fréquence d'une transition entre deux niveaux d'énergie d'un atome, ce qui constitue une référence stable et universelle. L'étalon qui définit actuellement la seconde est une transition entre deux niveaux hyperfins de l'atome de césium, située dans le domaine micro-onde, à $9 \mathrm{GHz}$. La précision des horloges à césium a connu une amélioration considérable, presqu'un facteur 100 , lorsque l'on a remplacé par des atomes froids les atomes d'un jet de césium se déplaçant à la vitesse thermique. La raison première est que des atomes refroidis peuvent être observés pendant un temps beaucoup plus long que les atomes d'un jet thermique. Cela permet d'obtenir des résonances atomiques plus fines d'un même facteur et, en conséquence, un pointé de ces résonances plus précis. L'autre raison essentielle tient au fait que le mouvement des atomes entraîne un changement apparent de la fréquence par effet Doppler, effet mécaniquement réduit avec des atomes froids. La figure 5 illustre l'amélioration progressive de la précision des horloges atomiques au cours du temps : on note le brusque progrès lié à l'utilisation des atomes froids en 1993. Les horloges à césium à atomes froids (fig. 6) atteignent aujourd'hui une exactitude relative de 3 à $41^{-16}$ !

Une nouvelle génération d'horloges, dites optiques, semble aujourd'hui amorcer une autre révolution, comme l'indiquent les résultats les plus récents portés sur la figure 5 . Les chercheurs développent de nouvelles horloges, utilisant d'autres atomes que le césium et basées sur des transitions atomiques dans le domaine optique et non plus microonde. La fréquence de ces transitions optiques est plus de dix mille fois plus élevée que celle de la transition d'horloge du césium! La plupart des effets limitant les performances des horloges étant indépendants en valeur absolue de cette fréquence, ils s'en trouvent réduits d'autant en valeur relative. Il a toutefois fallu résoudre plusieurs difficultés majeures pour que cette idée simple puisse être mise en pratique.

La première est qu'il n'existait pas d'électronique assez rapide pour compter les fréquences optiques, c'est-à-dire suivre les aiguilles de ces horloges à très haute fréquence. Ce problème est aujourd'hui résolu par l'utilisation de peignes de fréquence basés sur des lasers femtoseconde (voir l'article d'Anne Amy-Klein dans ce numéro).

La deuxième difficulté tient à l'effet Doppler mentionné plus haut. Celui-ci est indépendant en valeur relative de la fréquence de la transition, constituant la principale exception à la règle ci-dessus. Une solution est de confiner fortement les atomes. L'effet du mouvement résiduel des atomes dans le piège se réduit alors à l'apparition
5. Évolution de l'exactitude des horloges atomiques depuis les années 50 . En noir : les horloges à césium. On note la redéfinition de la seconde, à partir de la transition entre deux niveaux hyperfins de l'atome de césium 133 en 1967, puis l'amélioration significative apportée par les atomes froids (fontaines atomiques) à partir de 1993. En bleu : les horloges optiques avec l'espèce atomique utilisée. Depuis 2008, ces horloges sont les plus exactes : horloges à ions piégés $\left(\mathrm{Al}^{+}, \mathrm{Hg}^{+}\right)$, horloges à réseau optique (Sr, Yb).

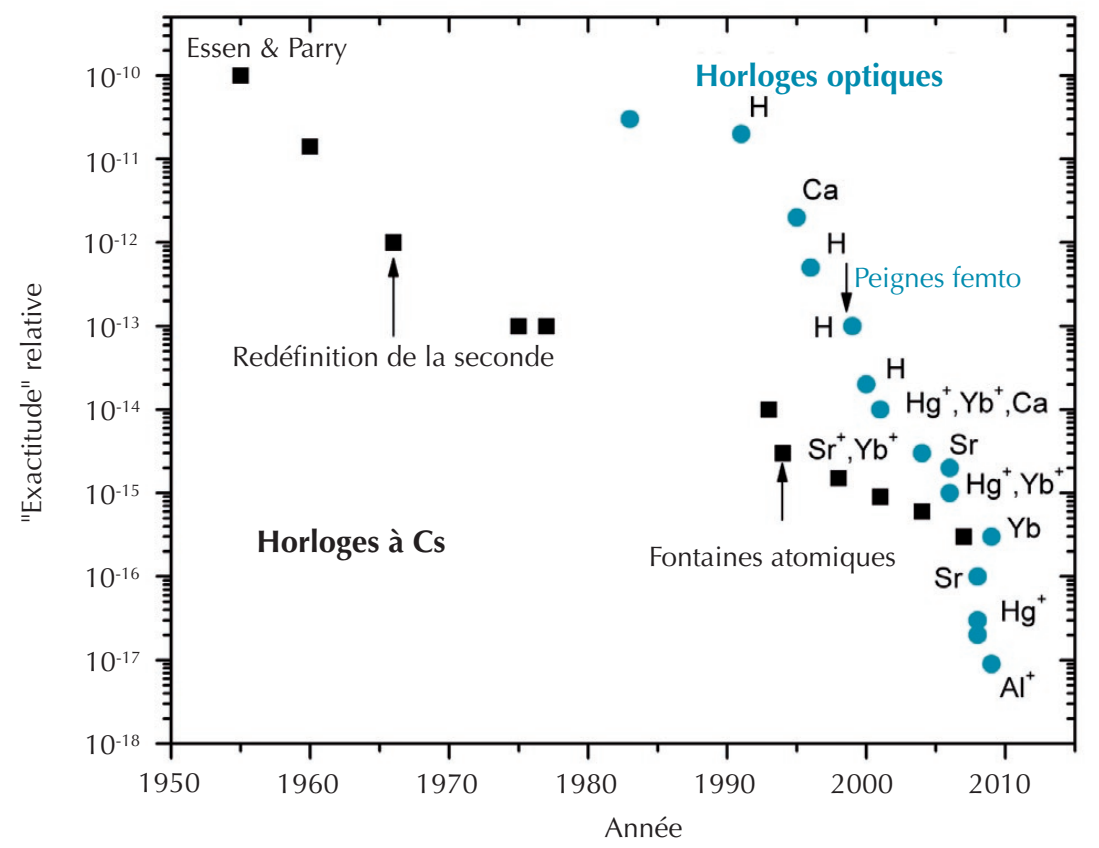


de résonances supplémentaires situées à des fréquences différentes de la résonance principale. Étant résolues (c'est-à-dire identifiables et séparables) en fréquence, ces résonances motionnelles ont un effet négligeable sur la transition d'horloge. Reste à réaliser un piège qui, tout en contraignant fortement le mouvement des atomes, ne modifie pas la fréquence de la transition de référence.

Cela est relativement facile à mettre en œuvre pour des ions, la charge de ces particules permettant le piégeage avec des champs extrêmement faibles. Les horloges à ions piégés constituent un domaine d'étude depuis les années 70 et sont depuis peu les horloges les plus exactes au monde, comme le montre la figure 5. Toutefois, la présence de cette charge entraîne des perturbations mutuelles de ces ions et, en pratique, ces horloges fonctionnent avec un ion unique. Or, disposer d'un grand nombre de particules de référence est hautement souhaitable, puisqu'on peut alors moyenner les signaux de ces particules et ainsi en principe réduire considérablement les fluctuations de fréquence de l'horloge. Il existe donc une motivation très forte pour utiliser des atomes neutres pour les horloges optiques.

On a vu plus haut comment piéger des atomes froids dans des réseaux optiques, mais les champs requis sont extrêmement intenses en comparaison de ceux utilisés pour les ions. Pour contourner ce problème, il a fallu attendre 2001 et la proposition par H. Katori de l'horloge à réseau optique. Celle-ci est basée sur une configuration très

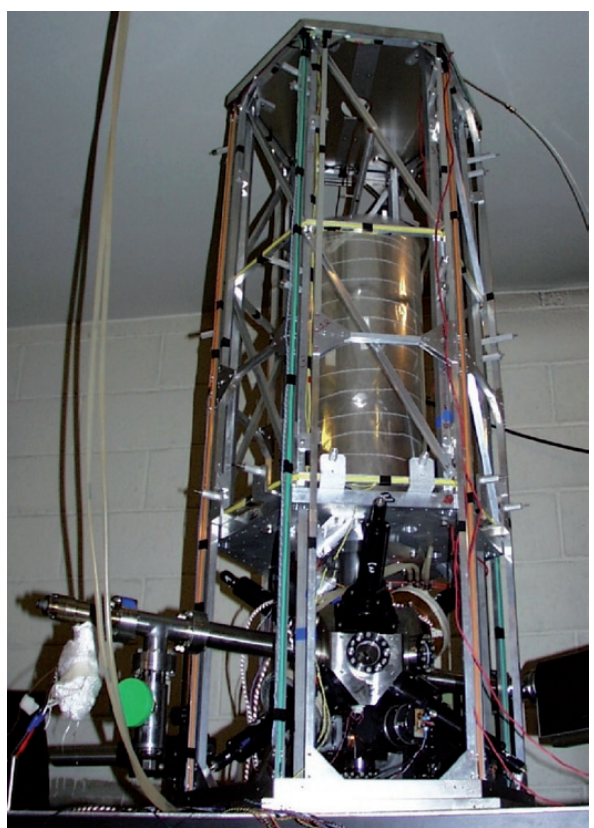

astucieuse où la perturbation due au piège est rigoureusement identique pour les deux niveaux d'énergie constituant la transition d'horloge. La fréquence de la transition entre ces niveaux n'est alors pas modifiée. Nous avons montré expérimentalement au SYRTE que les perturbations des deux niveaux sont identiques à $10^{-7}$ près, validant ainsi ce concept et ouvrant la voie à des horloges optiques à atomes froids dont l'exactitude atteindrait $10^{-18} \mathrm{en}$ valeur relative. Plus d'une dizaine de laboratoires à travers le monde développent ces horloges, avec divers types d'atomes froids.

Les meilleures horloges à réseau optique, celles du NIST et du JILA aux États-Unis et du SYRTE en France, sont d'ores et déjà les horloges les plus stables, tout en disposant encore d'une marge d'amélioration significative ! Leur exactitude surpasse celle des horloges à césium froid et s'approche des meilleures horloges à ions piégés. Une conséquence directe de ces progrès est qu'il sera inévitable à terme de redéfinir la seconde, le césium ne permettant plus d'atteindre les meilleures performances.

Il est intéressant de noter que ces horloges à atomes froids, application de recherches très fondamentales sur le contrôle quantique et l'interaction matière-rayonnement, constituent des outils de mesure sans égal pour nourrir en retour la recherche fondamentale. Un exemple en est l'étude des collisions entre atomes froids. Par la mesure de l'effet de ces interactions sur la fréquence d'horloge, on dispose d'informations

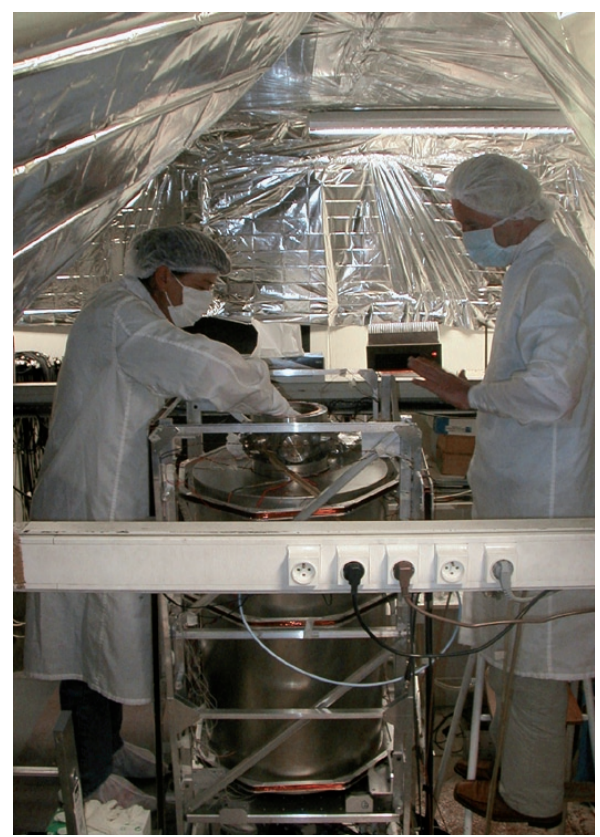

complémentaires de celles obtenues par l'étude de la dynamique de gaz quantiques dégénérés - amplitude de ces interactions, rôle des corrélations quantiques et de la nature bosonique ou fermionique des atomes, etc. Un autre exemple est donné par les tests du principe d'équivalence d'Einstein avec des horloges, complémentaires des tests d'isotropie de la vitesse de la lumière ou de l'universalité de la chute libre. Une manière de tester ce principe d'équivalence est de comparer de manière répétée des horloges basées sur des atomes différents, et de s'assurer que leur fréquence relative est une constante, indépendante du lieu et de l'instant où sont effectuées ces comparaisons.

Des tests fondamentaux plus riches encore sont envisageables si l'on est en mesure de les réaliser au moyen d'horloges dans l'espace ; ils offrent la possibilité d'explorer des conditions de potentiel gravitationnel très variées en orbite terrestre ou à l'échelle du système solaire, et de vérifier ainsi les prédictions de la relativité générale ou des théories cherchant à unifier la gravitation et les autres interactions fondamentales de la nature. À l'horizon 2014, une horloge à atomes froids de césium sera mise sur orbite à bord de la station spatiale internationale dans le cadre des projets PHARAO et ACES du CNES et del'Agence Spatiale Européenne. Pour une deuxième génération d'expériences à plus long terme, des études préliminaires sont d'ores et déjà en cours pour envoyer dans l'espace des horloges optiques à ions ou à atomes piégés.

(1) Le piège magnéto-optique combine des paires de faisceaux lasers se propageant en sens inverse et des gradients de champ magnétique.

(2) Ceci est une conséquence du principe de Pauli, qui interdit à deux fermions de même état de spin de se trouver au même endroit au même moment.

(3) Un isolant de Mott est un matériau qui devrait être conducteur dans le cadre de la théorie des bandes, mais qui se comporte comme un isolant lorsque les interactions répulsives entre particules sont suffisamment élevées pour les maintenir localisées sur les sites du réseau.

6. Horloge à fontaine de césium (SYRTE, Observatoire de Paris). Des atomes ultra-froids lancés verticalement sont interrogés deux fois par un signal micro-onde pendant leur trajet aller-retour sous l'effet de la gravitation. À gauche: vue générale de l'horloge. À droite : l'horloge en cours de modification. (Courtoisie: André Clairon et Roland Barillet, SYRTE.) 